\title{
Metagenomics for neurological infections - expanding our imagination
}

\section{Prashanth S. Ramachandran ${ }^{1,2}$ and Michael R. Wilson $\left(10^{1,2 \otimes}\right.$}

Abstract | Over the past two decades, the diagnosis rate for patients with encephalitis has remained poor despite advances in pathogen-specific testing such as PCR and antigen assays. Metagenomic next-generation sequencing (mNGS) of RNA and DNA extracted from cerebrospinal fluid and brain tissue now offers another strategy for diagnosing neurological infections. Given that $\mathrm{mNGS}$ simultaneously assays for a wide range of infectious agents in an unbiased manner, it can identify pathogens that were not part of a neurologist's initial differential diagnosis either because of the rarity of the infection, because the microorganism has not been previously associated with a clinical phenotype or because it is a newly discovered organism. This Review discusses the technical advantages and pitfalls of cerebrospinal fluid mNGS in the context of patients with neuroinflammatory syndromes, including encephalitis, meningitis and myelitis. We also speculate on how mNGS testing potentially fits into current diagnostic testing algorithms given data on mNGS test performance, cost and turnaround time. Finally, the Review highlights future directions for mNGS technology and other hypothesis-free testing methodologies that are in development.

"Physicians use heuristics or shortcuts in their decision making to help them sort through complex clinical information and formulate diagnoses efficiently"'. Because of the difficulites associated with diagnosing patients with complex neuroinflammatory syndromes such as encephalitis, myelitis and meningitis, neurologists are often forced to use cognitive heuristics and shortcuts when assessing these patients ${ }^{2}$. Indeed, many epidemiological studies have shown that an aetiological diagnosis is not obtained in $~ 50 \%$ of patients with encephalitis ${ }^{2,3}$. Improved diagnostic testing modalities are urgently needed to enhance individual patient care and to expand our understanding of the full spectrum of clinical phenotypes with which neurological infections can manifest ${ }^{4}$

Parallel revolutions in high-throughput sequencing and computational biology over the past two decades have yielded a new set of tools that are beginning to transform the way we approach the diagnosis (and exclusion) of neurological infections in patients with meningitis, myelitis and encephalitis. Metagenomic next-generation sequencing (mNGS) of cerebrospinal fluid (CSF) and brain biopsy tissue is a hypothesis-free approach to assay for a wide range of infections (DNA and RNA viruses, parasites, fungi and bacteria) in a single test. mNGS promises to fundamentally reorder the diagnostic algorithms for patients with suspected neurological infections $s^{5-17}$ (BOX 1).

The term 'metagenomics' refers to the interrogation of all the genetic material in an environmental sample. Enabled by the marked drop in the cost and increased speed of NGS technologies, the techniques for generating metagenomic data are evolving away from the amplification of pathogen-specific genes or genes conserved across many microbes to the use of millions of random primers that amplify virtually all of the nucleic acid in a sample.

Excellent publications already exist that describe in detail the various techniques by which extracted DNA and/or RNA can be prepared for $\mathrm{mNGS}^{18}$ as well as the numerous bioinformatics pipelines for analysing large mNGS datasets ${ }^{19-22}$. Given these publications and the rapidity with which the field is changing, this Review will not describe detailed laboratory or computational methods. Rather, we introduce some of the molecular and bioinformatics challenges associated with this technique to help neurologists understand the technical considerations and challenges that can affect the test's utility and the interpretation of results. In addition, we provide guidance to help neurologists and other subspecialists in internal medicine, infectious diseases, critical care and rheumatology decide whether and when to order 


\section{Key points \\ - Meningoencephalitis remains a challenging diagnosis owing to the multitude of possible infectious and autoimmune causes. \\ - Meningoencephalitis is associated with a high rate of morbidity and mortality and requires prompt diagnosis and treatment. \\ - Metagenomic next-generation sequencing (mNGS) is now a clinically validated test for neuroinfectious diseases that can aid clinicians with a timely diagnosis. \\ - mNGS can improve the detection of pathogens that were missed by clinicians or on standard direct testing. \\ - mNGS does not perform well when indirect tests are required to make the diagnosis (for example, serology), when infections are compartmentalized and for certain low abundance pathogens. \\ - The clinical context of the case is required when interpreting the results of mNGS.}

an mNGS test and how to interpret the significance of a positive or negative result. The Review highlights the ability of mNGS to look for the widest possible variety of organisms while also discussing its cost, along with computational and data interpretation challenges.

\section{Metagenomics for neurological infections}

Myriad reasons exist as to why the syndromes of encephalitis, meningitis and myelitis are frequently challenging to evaluate. The rarity of each of the many neuroinvasive pathogens makes physician-level knowledge of their overlapping clinical phenotypes poor and access to the individual diagnostic tests for these pathogens cumbersome. Indeed, diagnostic tests for amoebic infections, many arboviruses (viruses transmitted by arthropods), and rare bacterial and parasitic infections are only available through local departments of public health or national reference centres such as the Centers for Disease Control and Prevention. As a single test that can identify any neurological infection (except prions), mNGS can circumvent the need to order a huge number of pathogen-specific, candidate-based diagnostic tests (for example, pathogen-specific PCR, serology or antigen testing), each of which has its own strengths and weaknesses.

Immunocompromised patients present particular challenges because they are susceptible to unusual neuroinvasive pathogens that might not be part of a neurologist's standard diagnostic algorithm ${ }^{2,10,17,23-26}$. In addition, many emerging and re-emerging pathogens have neuroinvasive potential, including Ebola, measles, mumps, Nipah, Hendra, Chikungunya, Zika and Powassan viruses ${ }^{4,23,24,27-29}$. Zika virus had been circulating in Brazil for 18 months before it was identified and for 24 months before it was determined that it was responsible for a spike in cases of microcephaly and Guillain-Barré syndrome ${ }^{30}$. As it had not previously been seen in the Americas and had never been known to cause fetal brain abnormalities, it did not make sense for Brazilian physicians to test patients for Zika virus when these cases were first arising. As mNGS is an agnostic approach to identifying neurological infections, it has the potential to diagnose infections in patients with unexpected clinical phenotypes and/or demographics ${ }^{10,31-34}$ (FIG. 1, BOXES 2, 3).

Finally, the revolution in autoimmune neurology over the past 15 years has made it clear that many patients with previously assumed infectious encephalitis syndromes instead have autoantibody-mediated disorders. A 2018 study suggested that the prevalence of patients with autoimmune encephalitis might equal that of patients with infectious encephalitis ${ }^{35-38}$. The knowledge that patients with autoimmune encephalitis can respond favourably to powerful immunosuppressants $s^{39,40}$ has only made it more critical to identify (or exclude) an occult infection in a timely manner. To the extent that mNGS can help neurologists more confidently exclude an active CNS infection, mNGS has the potential to help speed the initiation of empirical immunosuppression in patients with suspected autoimmune encephalitis. Given the evolving diagnostic complexity and clinical severity of patients with meningoencephalitis, the management and diagnosis of patients with encephalitis is truly a multidisciplinary approach with input required from neurologists, infectious diseases specialists, neurointensivists, rheumatologists, immunologists, radiologists and microbiologists. mNGS is a powerful tool that fits into the overall diagnostic and management algorithm for these complex patients.

Identifying the optimal sample for mNGS. One of the most important considerations when deciding to pursue mNGS or when interpreting the significance of an mNGS result is to carefully assess the quality and timing of the available sample (or samples). mNGS is fundamentally a direct detection method, meaning that one is attempting to identify a pathogen by recovering its genomic DNA or RNA and/or transcriptional products. Thus, mNGS is susceptible to the same constraints as traditional, pathogen-specific PCR (that is, if the pathogen's nucleic acid is not physically present in the sample, then PCR and, by extension, mNGS will have no ability to detect it). As a result, patients with chronic infectious meningoencephalitis, generally considered as having symptoms for $>1$ month, might have a wider time window for obtaining a CSF sample that contains microbial nucleic acid ${ }^{5,10,11,34}$. However, for patients with acute viral encephalitis (for example, West Nile virus), the virus might only be present in the CNS for the first few hours or days of illness ${ }^{7,41}$. Thus, performing mNGS on CSF that is temporally remote from the onset of a patient's acute illness might not help identify the inciting infection (although it might increase confidence that infection is not ongoing in a patient who continues to suffer medical complications). Similarly, if a CSF sample has been stored at room temperature or even refrigerated at $4^{\circ} \mathrm{C}$ for multiple days before being tested, then the organism's nucleic acid (especially RNA) might have been degraded and mNGS might yield false negative results ${ }^{42}$.

A second important consideration is the patient's exposure to antimicrobials before the sample was obtained. Although pathogen-specific PCR and mNGS can detect residual microbial nucleic acid even after antibiotics have decreased the yield of culture ${ }^{14,43,44}$, a negative mNGS result needs to be interpreted with caution in this context. Finally, if a patient's infection is compartmentalized (for example, brain abscess) or if the suspected pathogen is typically diagnosed by serology 


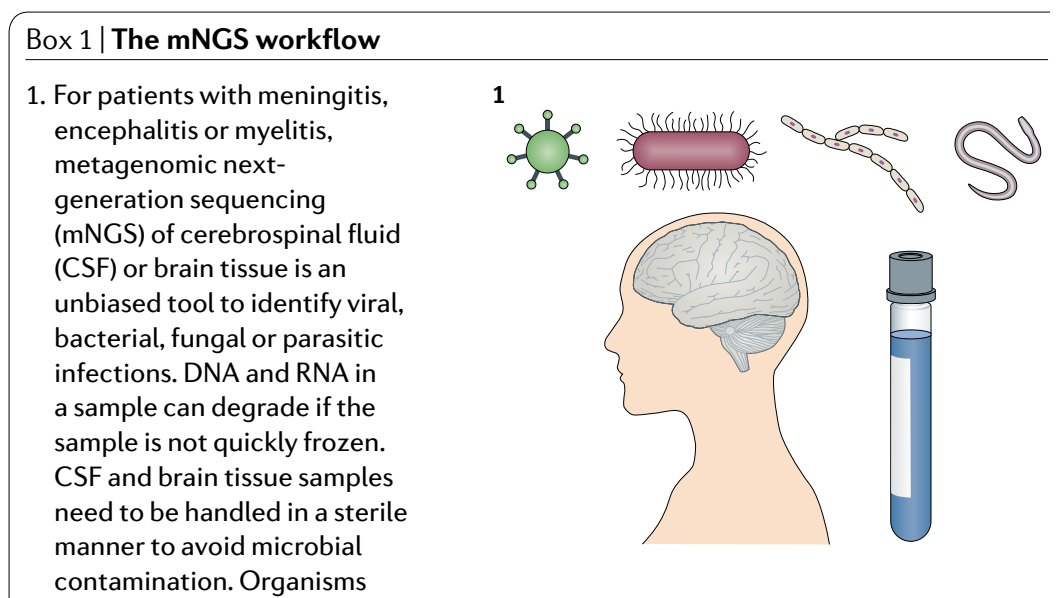

such as Treponema pallidum, whose DNA is hard to detect by PCR in CSF, will be similarly difficult to detect by mNGS.

2. RNA and DNA are extracted and randomly amplified (after reverse transcription of the RNA) to generate cDNA molecules for sequencing. A Clinical Laboratory Improvement Amendments (CLIA)-certified technician prepares samples with care to prevent environmental and cross-sample contamination. Enough sequences are generated to ensure adequate sensitivity for detecting non-human sequences against the human background.

3. The millions of sequences that are generated for each patient sample are analysed with a standardized and clinically validated bioinformatics pipeline. Sequences mapping to the human genome, typically comprising 97-98\% of CSF mNGS data, are removed. After additional quality filtering, non-human sequences are searched against large genetic databases to determine the best organism matches.

\section{A report is provided to the} clinician listing RNA or DNA viruses, parasites, fungi or bacteria that were detected above the reporting threshold. Organisms known to be frequent environmental contaminants are not reported. To place the mNGS findings in a clinical context, some testing centres provide ordering clinicians access to a clinical microbial sequencing board.
2

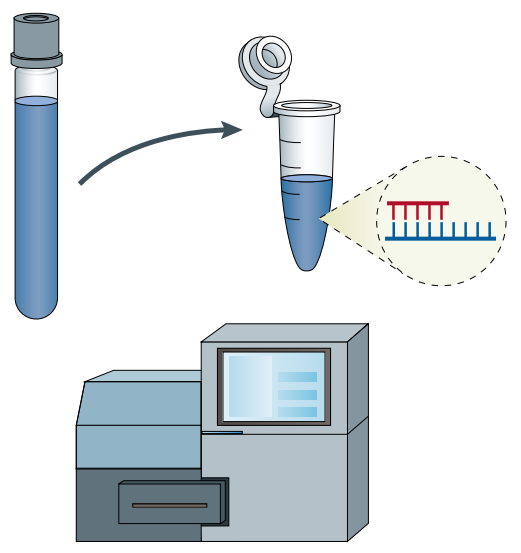

3

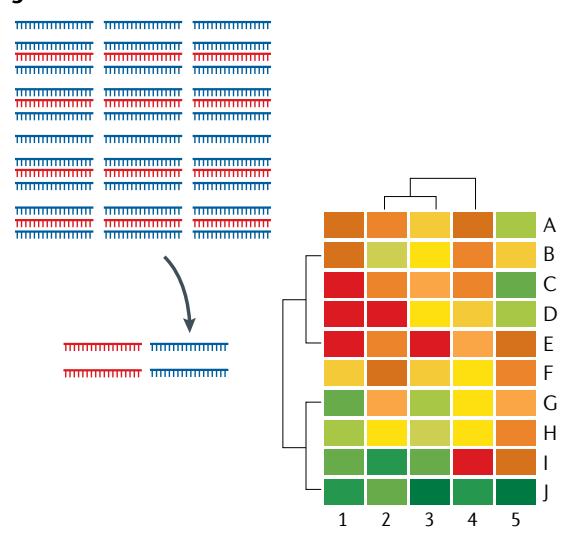

4

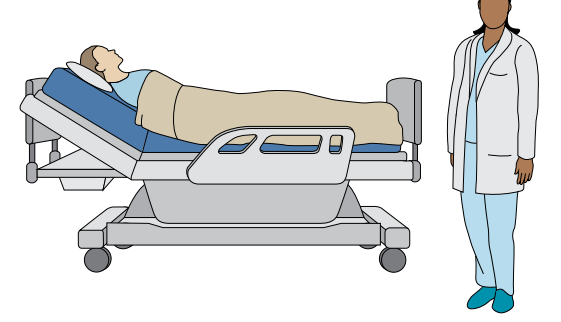

because of a low abundance or absence in the CSF (such as Borrelia burgdorferi (Lyme disease) or Treponema pallidum (syphilis)), a negative CSF mNGS result should be interpreted with caution ${ }^{14}$.

For these reasons, brain and/or meningeal tissue biopsy samples can also be valuable for interrogation by $\mathrm{mNGS}^{10,12,17,32,45-46,100}$. However, the success of this approach is dependent on whether the microorganism is present in the particular piece of tissue from which nucleic acid is being extracted, whereas CSF has the advantage of being a source of microbes from the whole subarachnoid space, if not the whole brain. Success is also dependent upon whether the tissue's nucleic acid (especially RNA) has been optimally preserved in a sterile manner. Flash freezing tissue in liquid nitrogen in the operating room avoids the degradation of nucleic acid and the environmental microbial contamination associated with formalin fixation and paraffin embedding as well as the microbial translocation from the gastrointestinal tract that can occur in the hours or days after a patient expires and before an autopsy is performed ${ }^{48}$.

Sequencing library preparation. After a sample is obtained, nucleic acid is extracted from $<1 \mathrm{ml}$ of the CSF sample (current clinically validated assays recommend at least $600 \mu \mathrm{l}$ but research-based sequencing has been performed with even smaller volumes) ${ }^{16,28}$. CSF can be a difficult sample type to perform mNGS on owing to its typically very low biomass ${ }^{28}$. Extracting nucleic acid from the CSF pellet after centrifugation might improve the detection of intracellular pathogens ${ }^{16,49}$. However, cellfree DNA from viruses might be more easily detected following extraction from the supernatant ${ }^{15}$. Detection of some pathogens, such as fungi and mycobacteria, is improved with enhanced extraction methods such as boiling and/or bead bashing ${ }^{15,50}$.

cDNA is generated from the RNA fraction by reverse transcription with random hexamer primers. The cDNA (or extracted DNA) is then converted into a library of random cDNA fragments with sequencing adapters ligated onto both ends of the cDNA molecules ${ }^{51}$. This pool of sequencing-competent cDNA molecules is then sequenced on a massively parallel scale by one of a number of available sequencing platforms (such as Illumina). Alternatively, high-throughput sequencing platforms that perform long-read sequencing on native RNA or DNA from a sample are increasingly available (for example, Pacific Biosciences and Oxford Nanopore). These platforms offer potential advantages, including the speed (that is, hours instead of days) at which a sample can be processed and sequenced (Oxford Nanopore) and the improved ability to assemble highly redundant microbial genomes from longer, intact stretches of nucleic acid $^{52-54}$. The Oxford Nanopore platform's flash drive size also makes it attractive for use in low-resource settings such as in a 2020 meningoencephalitis study performed in Vietnam ${ }^{55}$. More recent iterations of these long-read platforms show continued improvement, but their error rates continue to be higher than those of the short-read sequencing platforms such as Illumina ${ }^{56}$. This factor decreases their utility in detecting and diagnosing 
Prior knowledge combined with the patient history, physical examination, neuroimaging and initial laboratory studies form the backbone for the initial differential diagnosis.

Knowledge about clinical course and response to therapy is enhanced by a better understanding of the underlying aetiology and improves future patient evaluations.

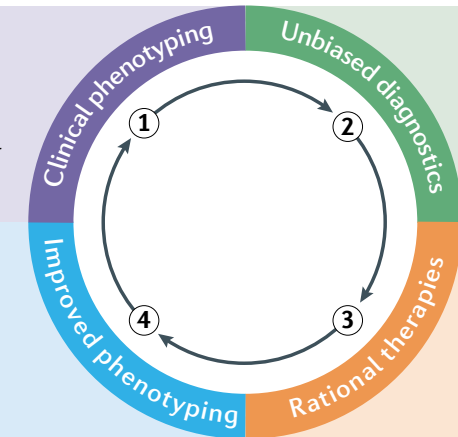

Metagenomic next-generation sequencing allows the neurologist to test for infections that are part of their differential diagnosis as well as for unanticipated infections.

A comprehensive approach to ruling in or ruling out infectious causes of meningitis and encephalitis enables more confident decisions about antimicrobial and/or anti-inflammatory therapies.

Fig. 1 | Virtuous learning cycle with hypothesis-free diagnostics. Unbiased metagenomic next-generation sequencing has the potential to identify rare and potentially unknown causes of meningoencephalitis, which can lead to rational therapeutic decision-making and to an improved understanding of the clinical spectrum with which various neurological infections can present.

infections in samples like CSF, which might contain only tens or hundreds of pathogen sequences with which to make a diagnosis.

Bioinformatics analysis. High-throughput sequencing technologies produce very large datasets. For example, Illumina-based mNGS protocols typically aim to generate 5-20 million 100-150 nucleotide (nt) sequences per sample. The delay in processing these massive amounts of data used to be a significant bottleneck preventing the delivery of clinically pertinent information in a timely manner. However, over the past few years, the time required to complete the initial data analysis has been reduced dramatically from weeks to 5-20 minutes ${ }^{5,14}$.

Conceptually, the many available bioinformatics pipelines for analysing mNGS data are similar in their need to filter out human, low complexity (that is, highly repetitive nucleotide sequences that are not likely to be informative for identifying a specific organism), redundant and poor-quality sequences before starting the process of determining the identity of the remaining non-human, high-complexity, non-redundant and high-quality sequences. The proportion of sequences that are removed by these filtering steps can vary considerably depending on the tissue type as well as on the abundance of the infectious agent and/or the degree of environmental contamination. For example, even in an infected CSF sample from a patient with encephalitis, $97-99 \%$ of the sequences might be human given the typically low pathogen loads, whereas $80 \%$ of the sequences from an infected sputum sample from a patient with viral pneumonia might be viral ${ }^{11,57}$.

Once the filtered mNGS dataset is obtained, a number of major bioinformatics decisions need to be made. First, one has to decide which database to use to identify the organisms to which the sequences best align. For example, the very large but error-prone National Center for Biotechnology Information's (NCBI) GenBank database contains genomic sequences from all known organisms, whereas some highly curated databases only contain high-quality genomic information from known human pathogens ${ }^{11,12,14,16,20,58-60}$. Using the GenBank database increases the likelihood of identifying more unusual or divergent infections but requires the analyst to perform secondary analyses to confirm that a preliminary organism match is correct and not the result of an erroneous entry in the database. Using other, more limited, databases makes it less likely that an initial microbial call is erroneous, but it is also less likely that an infectious agent not contained in the more limited dataset will be identified. Second, bioinformatics pipelines differ on whether to first assemble the typically short sequencing reads (100-200 nt) into larger contigs for more specific organism matching or whether to do an initial search with the raw, short sequences. Finally, decisions need to be made about the relative weight to place on the nucleotide-to-nucleotide matches, which are more stringent and less tolerant of organisms with divergent genome sequences than nucleotide-to-amino acid matching; the latter is more sensitive to the detection of divergent organisms but also more likely to generate spurious matches ${ }^{18}$.

Data interpretation. Once the microbial identifications have been made from the filtered dataset, the primary task is to determine which, if any, of these microbes represent an infectious agent (or agents) and what proportion represent contaminants (for example, from skin flora or laboratory reagents) that are omnipresent in mNGS datasets.

Although CSF is a sterile bodily fluid, contaminating microbial sequences are ironically a major problem for CSF. As previously discussed, pathogen loads are typically low in CSF and, therefore, there are usually very few sequences that align to the infectious agent. In addition, we and others have shown that, in very low biomass samples like CSF (typical CSF RNA inputs are 5-50 pg), an overamplification of sequencing reagent contaminants occurs ${ }^{11,28,61,62}$. In other words, when little to no biomass exists in a CSF sample, the primers used in the PCR amplification step amplify even minimal quantities of environmental contaminants over and over, thus increasing the proportional representation of contaminants in the final dataset. Fortunately, the addition of even 20 pg of RNA of a known sequence can substantially decrease the overrepresentation of sequencing reagent contaminants without sacrificing sensitivity for detecting an infectious organism ${ }^{11}$. Similarly, observing that an organism's representation in a dataset is inversely 
correlated to the input RNA amount makes it likely that this organism is a reagent contaminant ${ }^{61,62}$.

Another critical component for differentiating between infections and contaminants is the use of 'no template' (that is, sterile water) and uninfected CSF controls to characterize the microbes present in a particular laboratory as well as the DNA and RNA from skin flora that frequently contaminate CSF obtained by lumbar puncture. With these data, one can construct background models and use a variety of scoring metrics (for example, Z-score based or absolute cut-offs) to determine how unexpected it is to find a particular organism in a given patient's sample based on its abundance across uninfected CSF (or brain) samples and water (that is, no template) controls ${ }^{11,16}$.

As with all clinical test results, the potential pathogens identified by mNGS must be put into clinical context to determine whether they are clinically relevant. To facilitate this contextualization, in our institute, we offer 'clinical microbial sequencing boards' attended by neurologists, infectious diseases experts, laboratory medicine specialists and scientists with expertise in mNGS, during which the details and implications of the mNGS results and analyses (including secondary analyses discussed below) can be discussed in the context of the treating physician's understanding of the clinical features of the case $^{14}$. For example, Case 4 (BOX 3) highlights a case in which Epstein-Barr virus was detected in CSF by PCR but was not ultimately the aetiological agent of the

\section{Box 2 | Identifying pathogens omitted during conventional work-up}

\section{Case 1}

A 41-year-old woman had chronic and recurrent meningitis for 15 years. At presentation, owing to risk factors for tuberculosis, she was treated for tuberculous meningitis. Despite treatment, she had recurrent episodes of meningitis and developed lumbar arachnoiditis with a persistent cerebrospinal fluid (CSF) lymphocytic pleocytosis and hypoglycorrhachia. MRI demonstrated lumbar arachnoiditis with a transient cyst-like structure in the lumbosacral sac. A multitude of infectious aetiologies were tested for, but all tests returned negative results. It was therefore felt that she probably had an autoimmune neurological condition and was treated with long-term immunosuppression. This treatment successfully reduced her level of CNS inflammation but she developed steroid-dependency, with flares of meningitis whenever her steroid dose was reduced. Metagenomic next-generation sequencing (mNGS) detected sequences to Taenia solium (pork tapeworm), which was confirmed with orthogonal antigen and serology testing. She was commenced on dual anti-helminthic therapy with an excellent response ${ }^{34}$.

\section{Case 2}

A 26-year-old woman with an initially undisclosed history of intravenous drug use (IVDU) presented with a 1-year history of low back pain and 4 days of saddle anaesthesia and a left foot drop. MRI of the lumbar spine displayed diffuse leptomeningeal enhancement with a loculated rim enhancing collection compressing the conus medullaris. The CSF displayed a neutrophilic pleocytosis and multiple investigations, including $16 \mathrm{~S}$ and $18 \mathrm{~S}$ universal RNA PCR and tissue biopsy, yielded negative results. Over the course of several months, her symptoms progressed to the point that she was wheelchair bound. mNGS detected Candida dubliniensis, which was consistent with the history of IVDU that she disclosed upon additional questioning. She was treated with antifungals with an excellent outcome ${ }^{11}$

In Case 1, the patient had a common neurological infection (neurocysticercosis) that presented in an atypical manner. She only developed a solitary and short-lived subarachnoid cyst years into her chronic and recurrent meningitis, and it did not raise suspicion for neurocysticercosis. In Case 2, the patient's ultimate diagnosis raised suspicions for a history of IVDU (which were later confirmed) given the epidemiology of Candida meningitis in immunocompetent adults. patient's meningoencephalitis ${ }^{63}$. Human herpesvirus type 6 is another common example of a virus that can cause meningoencephalitis in patients who have undergone bone marrow transplantation ${ }^{64}$; however, more often, it is thought to be a bystander virus.

Of note, the abundance of sequencing reads aligned to a presumed pathogen has a gross correlation with the abundance of the pathogen in the CSF; however, this correlation is not a true linear correlation and can vary on the basis of several factors such as RNA degradation, sample extraction techniques and PCR amplification bias $^{16}$. Although serial mNGS studies could be used to document the resolution of an infection, it would be more cost-effective to track this in subsequent CSF samples with a pathogen-specific quantitative PCR assay if one is available.

Secondary analyses. Beyond the identification of a particular infection, mNGS datasets permit a wide variety of secondary analyses. For example, enough of an organism's genome might be recovered from an mNGS dataset to be able to perform phylogenetic analyses that can help determine the time and place where a patient was infected $^{14,65}$, identify whether antimicrobial resistance genes are present and to determine whether a particular patient's infection might be connected to a wider disease outbreak in the hospital or their geographic region $^{14,65-68}$. Although the results of these secondary analyses might not be part of the official clinical report for a clinical mNGS test, they can be discussed with the treating physicians (for example, in the context of a clinical microbial sequencing board) and inform additional diagnostic testing or even public health responses ${ }^{14}$.

\section{Enrichment and depletion technologies}

The depth of sequencing (that is, how many individual sequences are obtained for an individual sample) is an important consideration as samples can have low pathogen loads and/or a high number of human sequences (for example, owing to a high CSF pleocytosis) or environmental background contamination. Thus, increasing the sequencing depth is a potential solution to improve the sensitivity of an mNGS test. This approach is becoming more feasible as sequencing capacity increases and the price per nucleotide sequenced drops rapidly. In addition, a variety of novel targeted depletion and enrichment technologies that can increase the diagnostic yield without needing to increase the sequencing depth have been developed over the past few years.

Depletion. We and others have found that human transcripts in CSF mNGS RNA-Sequencing datasets can be heavily skewed towards mitochondrial and ribosomal RNA genes, sometimes representing 50-80\% of all sequences in a sample ${ }^{69}$. Thus, targeted depletion of this relatively small number of highly expressed, human RNA transcripts could theoretically enhance detection of infections while lowering sequencing costs. Although numerous human ribosomal and mitochondrial depletion kits are commercially available, they require multiple nanograms of input RNA and are thus not useful for the very low RNA yields from CSF. DASH (Depletion 


\section{Box 3 | Identifying pathogens missed on conventional testing}

\section{Case 3}

A 60-year-old man with a history of follicular lymphoma on maintenance rituximab presented with 1 week of orchiepididymitis followed 3 days later by a cerebellar syndrome, with investigations demonstrating a modest lymphocytic cerebrospinal fluid (CSF) pleocytosis. He had a rapid deterioration in his level of consciousness and an MRI of the brain showed diffuse cerebellar oedema, obstructive hydrocephalus, diffuse leptomeningeal enhancement, and periventricular, thalamo-mesencephalic and basal ganglia T2-weighted signal abnormalities. He had an extensive infectious work-up and died despite several empirical therapies. Days after his death, Powassan virus IgM ELISA was negative. Metagenomic next-generation sequencing (mNGS) detected reads to Powassan virus and was also able to strain type the pathogen to the deer tick virus lineage II, which was confirmed with RT-PCR ${ }^{100}$.

\section{Case 4}

A 14-year-old girl on chronic immunosuppression for a renal transplant presented with meningoencephalitis after a camping trip in Angeles National Forest, California. The CSF displayed a neutrophilic pleocytosis and multiple infectious investigations returned negative, including West Nile virus (WNV) IgM in serum and CSF. A brain MRI showed bilateral thalamic $\mathrm{T} 2$ hyperintensities. She was started on broad-spectrum antibiotics and, when there was no evidence of improvement, she was treated with intravenous immunoglobulin for possible acute disseminated encephalomyelitis. Epstein-Barr virus was detected in her CSF by PCR and, as no other cause was found, she was treated with ganciclovir. mNGS later detected WNV RNA, confirmed with convalescent WNV serology. The patient's clinical presentation was consistent with neuroinvasive WNV disease and not with Epstein-Barr virus encephalitis. The patient improved in time with supportive care ${ }^{7}$.

Both cases illustrate the utility of direct detection methods such as mNGS in immunocompromised patients with acute encephalitis syndromes. The patients' immunosuppressed states probably increased the yield of mNGS as the WNV and Powassan virus infections were probably present in the CSF at higher titres and for longer periods of time, while also decreasing the sensitivity of the acute viral serologies that are typically used to diagnose these infections. of Abundant Sequences by Hybridization) is a targeted and programmable tool that removes unwanted host sequences and is agnostic to the input sample type and amount ${ }^{69}$. After generating cDNA from the input RNA, DASH uses CRISPR-Cas9 to selectively target and cut DNA molecules that are complementary to guide RNA sequences, thus rendering these DNA molecules unsuitable for final sequencing library amplification and sequencing ${ }^{69}$. Effective depletion strategies for human DNA that are compatible with mNGS workflows and that result in significant levels of pathogen sequence enrichment have proven more challenging given that DNA samples generate much more evenly distributed coverage across the human genome and, therefore, selective depletion of a finite number of genes does not substantially enhance the detection of non-human sequences.

Enrichment. Certain pathogens can be in low abundance and, despite being detected, might be below the clinical reporting threshold for a given mNGS assay. Mycobacterial infections in particular are challenging in this regard given that tuberculous meningitis is a paucibacillary infection (that is, low numbers of bacilli are needed to cause infection) ${ }^{14}$. Several methods are currently used to enrich low abundance organisms. VirCapSeq-VERT (Virome Capture Sequencing Platform for Vertebrate Viruses) and related methods can enrich for viral sequences by up to 10,000 -fold ${ }^{70,71}$. In VirCapSeq-VERT, 2 million oligonucleotide probes designed to bind to the coding site of all viral taxa known to infect vertebrae are hybridized to a cDNA library. Once added to a sample, these probes attach to complementary viral DNA. Streptavidin magnetic beads are added to the probes and their associated cDNA components. The beads are magnetically captured and cDNA is removed, followed by post-hybridization PCR and sequencing.

FLASH (Finding Low Abundance Sequences by Hybridization) is a novel enrichment method that utilizes CRISPR-Cas9 technology ${ }^{72}$. Prior to library preparation, DNA is dephosphorylated using calf intestinal alkaline phosphatase, rendering any exposed $5^{\prime}$ ends inaccessible to adaptor ligation. This prevents adaptor ligation to the majority of the sample, including host and non-host nucleic acids. Guide RNAs are then added to direct Cas9 to cut DNA at predefined targets, which allows the newly exposed DNA to undergo adaptor ligation. FLASH has enriched targeted sequences by $>100,000$ fold in initial studies with Plasmodium falciparum and Staphylococcus aureus ${ }^{72}$. Although FLASH deviates from the unbiased approach of mNGS, it might have utility as an adjunct test in cases with high suspicion for specific low abundance pathogens.

Metagenomic sequencing with spiked primer enrichment is another method that enables the targeted amplification of specific pathogen sequences combined with the unbiased advantages of $\mathrm{mNGS}^{73}$. In an initial study, primers targeting 15 virus genomes were spiked in along with the random primers used for mNGS library construction. These spiked primers amplified their specified viral genomes at a median ten-fold enrichment. The improved detection of specific viral pathogens at lower sequencing depths, the maintenance of the unbiased approach of mNGS, the additional cost of only US $\$ 0.34$ per sample and no additional time required for the mNGS protocol, make this an appealing enrichment technique ${ }^{73}$. Panels for specific infections can be custom designed for the unique patient cohorts being tested and/or for pathogens known to only be present at low abundance.

\section{Clinical evaluation and adoption}

Relegated to research labs for many years, CSF mNGS testing is now clinically available (at the time of writing, UCSF Clinical Laboratory is the only provider of the assay for CSF in the United States, but other laboratories have assays at different stages of development, with Johns Hopkins Medicine soon to launch a clinical assay $\left.{ }^{15}\right)$. Although not meant to be an exhaustive list, internationally, clinical CSF mNGS testing is available in the United Kingdom ${ }^{74}$, France ${ }^{75}$, South Korea ${ }^{54}$ and China ${ }^{76,77}$. The validation data for the UCSF assay demonstrated that it had a sensitivity of $73-92 \%$ and a specificity of $96-99 \%$, depending on the pathogen ${ }^{16}$. These results were based on comparative testing of 73 known positive and 22 negative CSF samples followed by testing of a further 20 cases with 12 known positive samples. After the test was validated, a multicentre study was performed to evaluate its real-world performance ${ }^{14}$. The Precision Diagnosis of Acute Infectious Diseases (PDAID) study enrolled 204 patients with idiopathic 
meningitis, encephalitis or myelitis at eight hospitals and found that $\mathrm{mNGS}$ had $80 \%$ positive percent agreement with infections identified by any direct detection method on CSF (that is, culture, antigen testing, PCR and orthogonally confirmed mNGS) and $98 \%$ negative percent agreement. As mNGS identified 13 infections missed by standard testing, conventional CSF direct detection tests only had $67.5 \%$ positive percent agreement and $99.4 \%$ negative percent agreement relative to infections identified by direct detection methods on CSF, including orthogonally confirmed mNGS. Overall, mNGS of CSF increased the infectious diagnoses by $22 \%$ in the PDAID study. Of the 13 cases diagnosed only by mNGS, 8 diagnoses had an effect on clinical decision-making.

Although mNGS had good concordance with other direct detection methods on CSF and, indeed, increased the overall diagnostic yield, it did not detect 26 (45\%) of the total infections diagnosed in the PDAID study. As discussed previously, there were three reasons why an infection was missed. Of the 26 infections, 11 were diagnosed by serology alone (for example, West Nile virus and T. pallidum); in these cases, both mNGS and the pathogen-specific PCR were concordant in not finding evidence for the pathogen's nucleic acid in the CSF. Furthermore, seven infections were compartmentalized (for example, brain abscess) and were identified by sampling tissue other than CSF; again, with these infections, the negative results from CSF mNGS were concordant with pathogen-specific PCR on CSF. Finally, eight infections were true false negatives by mNGS as low titres of microbial DNA were detected by pathogen-specific CSF PCR, but the sequences to the infectious agent identified by mNGS were either not abundant enough to reach the reporting threshold for the mNGS assay $(n=6$; Mycobacterium bovis, M. tuberculosis, Cryptococcus neoformans, Propionibacterium acnes, fusobacterium, $S$. aureus) or had no reads detected on mNGS $(n=2$; cytomegalovirus and herpes simplex virus type 2 ). In three cases, mNGS results were found to be false positives after additional discrepancy testing was performed (Pantoea, S. aureus and Streptococcus agalactiae). The false positives were attributed to sample contamination from the environment or normal human flora. These findings again highlight that clinical reasoning should still be used to interpret test results and to order serological tests and/or tests on other relevant tissue types when appropriate.

\section{Practical considerations for $\mathbf{m N G S}$}

Although compelling individual cases from the PDAID study and other case reports and case series suggest that mNGS might lead to improved health outcomes and potential cost savings to the health-care system, the PDAID study did not include a control group of patients for whom mNGS testing was not offered. Thus, it could not answer important questions about the patient populations for whom CSF mNGS testing will be most cost-effective, when mNGS should be utilized in the course of a patient's care and whether, at a population-level, mNGS improves health outcomes for patients with meningitis, encephalitis or myelitis.
The few available clinically validated mNGS assays on blood, CSF and respiratory fluid range in cost from US $\$ 1,000$ to $\$ 2,500$ and test turnaround times range from 1 to 10 days. The variable turnaround times are not due as much to technical variations in the assays but rather to staffing levels and the degree of automation, both of which will increase as these tests become more common and routine ${ }^{18}$. A health-care economics modelling study based on actual insurance payments (as opposed to amounts charged) for hospitalized patients with meningitis or encephalitis found that an opportunity exists for mNGS testing to be cost-effective in patients who have undergone a neurosurgical procedure, who are critically ill, who are infected with HIV or who have had a solid organ transplant given that these patients have long lengths of stay and substantial costs throughout the length of their hospitalization ${ }^{78}$. For example, mNGS could decrease health-care costs by diagnosing a treatable disease, as seen in a patient who had a lung transplant and was diagnosed with hepatitis $\mathrm{E}$ virus meningoencephalitis and whose anti-viral treatment resolved their neuroinflammatory disease and probably also spared them a liver transplant ${ }^{9}$. In other cases, the diagnosis of even a fatal infection can save health-care costs by allowing families and doctors to focus on palliative care rather than on additional diagnostic testing, empirical treatments and costs associated with critical care ${ }^{8}$.

Cost considerations aside, the decision about when in the course of a patient's work-up to order a CSF mNGS test is ultimately one that has to be made on a case-by-case basis. Factors include the physician's suspicion for an unusual infection not easily identified by available conventional tests and the quality of available CSF samples as judged by the timing of their collection relative to symptom onset and whether the sample has been adequately handled to preserve sterility and nucleic acids.

\section{Future directions}

Two new frontiers are beckoning in the field of hypothesis-free testing. As discussed above, host transcriptomic data constitute the bulk of the mNGS data generated from sequencing CSF RNA. In similar datasets from the blood of patients with sepsis and respiratory samples from patients with a variety of infectious and non-infectious causes of pneumonia, host gene expression signatures can correctly classify patients as having infectious or non-infectious syndromes and even distinguish between patients with particular classes of infections (for example, bacterial versus viral) ${ }^{57,79-83}$. Parallel efforts are underway to develop syndromic classifiers from CSF RNA-sequencing data (which are already generated as part of existing mNGS assays). These classifiers could have important implications for clinical management. For example, in addition to not finding an infection in the CSF of a patient with suspected autoimmune encephalitis, it might increase the treating physician's confidence to embark on a course of empirical immunosuppression if they know that the patient's host response mirrors that seen in other patients with autoimmune encephalitis compared to patients with viral encephalitis. 


\section{Box 4 | Pros and cons of metagenomic next-generation sequencing}

Pros

- Single test that can diagnose infections from fungi, DNA and RNA viruses, bacteria and parasites

- Can identify emerging pathogens that are either novel to the region or highly divergent from known pathogens

- Can identify common infections presenting in an atypical manner or overlooked by the treating team

- Clinically validated assays are increasingly available

Cons

- Expensive: current costs of the clinical assay are $\sim$ US $\$ 2,000$

- Dependent on the presence of microbial nucleic acid; therefore, it is insensitive for compartmentalized or transient infections

- Can be insensitive for low titre ( $<100$ copies) infections or with high human DNA or RNA background (for example, pleocytosis $(\sim 500-1,000$ cells $/ \mu \mathrm{l})$ )

- Environmental contamination might lead to false positives; the clinical context and appropriateness of the result should always be considered

Secondly, powerful technologies are emerging to comprehensively survey the CSF (and other bodily fluids) for antibodies to a large number of viruses and autoantibodies. Perhaps most prominently, programmable phage display assays (such as VirScan) that display tens or hundreds of thousands of viral peptides on the surfaces of a library of T7 bacteriophages can generate serological evidence for a neuroinvasive viral infection even when the viral nucleic acid is no longer present ${ }^{84-87}$.

CRISPR-Cas systems are also being developed for the direct detection of pathogen sequences in clinical samples without needing to extract DNA or RNA, let alone perform any amplification or sequencing steps. The most prominent two methods are SHERLOCK using CRISPR-Cas13 $\left(\right.$ REFS $\left.^{88,89}\right)$ and DETECTR using CRISPR-Cas $12 \mathrm{a}^{89-91}$. Assays have already been developed to detect Zika, dengue, West Nile, yellow fever and human papilloma viruses as well as severe acute respiratory syndrome coronavirus type 2 (REFS ${ }^{88-90,92,93}$ ). Although these techniques are targeted and do not permit an unbiased assessment of the nucleic acid in a sample, they can be increasingly multiplexed ${ }^{89,93}$, generate a fluorescent signal with no computational analysis required, generate an answer in $<2$ hours and can be performed with lyophilized reagents that are conducive to diagnostic testing in low-resource settings ${ }^{90}$.

\section{Conclusions}

The diagnosis of neurological infections through the detection of microbial nucleic acid in CSF began with the advent of herpes simplex virus PCR, which transformed the diagnosis of herpes simplex encephalitis from requiring a brain biopsy to a diagnosis that could be made from CSF in a matter of hours ${ }^{94}$. Subsequently, multiplex PCR panels that assay for 10-20 infections in parallel ${ }^{95}$ as well as more broad-based PCR strategies that amplify highly conserved regions like $16 \mathrm{~S}$ ribosomal RNA (rRNA), 18S rRNA or 28S rRNA of bacterial, fungal or parasitic genomes, respectively ${ }^{41,96-99}$, have further advanced PCR-based diagnostics for neurological infections. Now that NGS data are increasingly cheap and easy to acquire and analyse, $\mathrm{mNGS}$ represents the next step in an increasingly unbiased approach to diagnosing neurological infections, and it is one of the most exciting translational applications of the genomics revolution for neurologists.

We have discussed the prospects for mNGS and other related genomic technologies to improve the landscape of the diagnosis of neurological infections and our understanding of neuroinflammatory disorders more generally. The unbiased nature of mNGS will help combat some of the cognitive heuristics or shortcuts that neurologists rely on when evaluating a complex clinical case with incomplete information and open up our imagination to the diverse ways in which pathogens can manifest as disease, especially when they interact with that most complex of human organs, the brain. However, a thorough understanding of the strengths and weaknesses of these technologies is important to mitigate against falling prey to another cognitive shortcoming, namely blind obedience to technology (BOX 4). Even results from advanced diagnostic testing must be interpreted in the clinical context of a patient from whom a physician has obtained a thorough history and examination.

Published online 13 July 2020
1. Vickrey, B. G., Samuels, M. A. \& Ropper, A. H. How neurologists think: a cognitive psychology perspective on missed diagnoses. Ann. Neurol. 67, 425-433 (2010).

2. Glaser, C. A. et al. In search of encephalitis etiologies: diagnostic challenges in the California Encephalitis Project, 1998-2000. Clin. Infect. Dis. 36, 731-742 (2003).

3. Granerod, J. et al. Challenge of the unknown. A systematic review of acute encephalitis in nonoutbreak situations. Neurology 75, 924-932 (2010).

4. Nath, A. Neuroinfectious diseases: a crisis in neurology and a call for action. JAMA Neurol. 72, 143-144 (2015).

5. Wilson, M. R. et al. Actionable diagnosis of neuroleptospirosis by next-generation sequencing N. Engl. J. Med 370, 2408-2417 (2014).

6. Wilson, M. R. et al. Diagnosing Balamuthia mandrillaris encephalitis with metagenomic deep sequencing. Ann. Neurol. 78, 722-730 (2015).

7. Wilson, M. R. et al. Acute west nile virus meningoencephalitis diagnosed via metagenomic deep sequencing of cerebrospinal fluid in a renal transplant patient. Am. J. Transpl. 17, 803-808 (2017).

8. Chiu, C. Y. et al. Diagnosis of fatal human case of St. Louis encephalitis virus infection by metagenomic sequencing, California, 2016. Emerg. Infect. Dis. 23 1964-1968 (2017).

9. Murkey, J. A. et al. Hepatitis E virus-associated meningoencephalitis in a lung transplant recipient diagnosed by clinical metagenomic sequencing. Open. Forum Infect. Dis. 4, of 121 (2017).

10. Wilson, M. R. et al. A novel cause of chronic viral meningoencephalitis: Cache Valley virus. Ann. Neurol. 82, 105-114 (2017)

11. Wilson, M. R. et al. Chronic meningitis investigated via metagenomic next-generation sequencing. JAMA Neurol. 75, 947-955 (2018).

12. Salzberg, S. L. et al. Next-generation sequencing in neuropathologic diagnosis of infections of the nervous system. Neurol. Neuroimmunol. Neuroinflamm 3, e251 (2016).

13. Piantadosi, A. et al. Rapid detection of Powassan virus in a patient with encephalitis by metagenomic sequencing. Clin. Infect. Dis. 66, 789-792 (2018).

14. Wilson, M. R. et al. Clinical metagenomic sequencing for diagnosis of meningitis and encephalitis. N. Engl. J. Med. 380, 2327-2340 (2019).

15. Simner, P. J. et al. Development and optimization of metagenomic next-generation sequencing methods for cerebrospinal fluid diagnostics. J. Clin. Microbiol. 56, e00472-18 (2018).
16. Miller, S. et al. Laboratory validation of a clinical metagenomic sequencing assay for pathogen detection in cerebrospinal fluid. Genome Res. 29, 831-842 (2019).

17. Morfopoulou, S. et al. Deep sequencing reveals persistence of cell-associated mumps vaccine virus in chronic encephalitis. Acta Neuropathol. 133, 139-147 (2017).

18. Chiu, C. Y. \& Miller, S. A. Clinical metagenomics. Nat. Rev. Genet. 20, 341-355 (2019).

19. Naccache, S. N. et al. A cloud-compatible bioinformatics pipeline for ultrarapid pathogen identification from next-generation sequencing of clinical samples. Genome Res. 24, 1180-1192 (2014).

20. Wood, D. E. \& Salzberg, S. L. Kraken: ultrafast metagenomic sequence classification using exact alignments. Genome Biol. 15, R46 (2014).

21. Flygare, S. et al. Taxonomer: an interactive metagenomics analysis portal for universal pathogen detection and host mRNA expression profiling. Genome Biol. 17, 111 (2016).

22. Ramesh, A. et al. Metagenomic next-generation sequencing of samples from pediatric febrile illness in Tororo, Uganda. PLoS One 14, e0218318 (2019). 
23. Tyler, K. L. Emerging viral infections of the central nervous system: part 2. Arch. Neurol. 66, 1065-1074 (2009).

24. Tyler, K. L. Emerging viral infections of the central nervous system: part 1. Arch. Neurol. 66, 939-948 (2009).

25. Palacios, G. et al. A new arenavirus in a cluster of fatal transplant-associated diseases. N. Engl. J. Med. 358 , 991-998 (2008)

26. Pérot, P. et al. Identification of Umbre Orthobunyavirus as a novel zoonotic virus responsible for lethal encephalitis in 2 French patients with hypogammaglobulinemia. Clin. Infect. Dis. https:// doi.org/10.1093/cid/ciaa308 (2020).

27. Wilson, M. \& Tyler, K. L. Emerging diagnostic and therapeutic tools for central nervous system infections. JAMA Neurol. 73, 1389-1390 (2016).

28. Saha, S. et al. Unbiased metagenomic sequencing for pediatric meningitis in Bangladesh reveals neuroinvasive chikungunya virus outbreak and other unrealized pathogens. mBio 10, e02877-19 (2019).

29. Howlett, P. J. et al. Case series of severe neurologic sequelae of Ebola virus disease during epidemic, Sierra Leone. Emerg. Infect. Dis. 24, 1412-1421 (2018).

30. Faria, N. R. et al. Zika virus in the Americas: early epidemiological and genetic findings. Science 352 , 345-349 (2016)

31. Quan, P. L. et al. Astrovirus encephalitis in boy with X-linked agammaglobulinemia. Emerg. Infect. Dis. 16 918-925 (2010)

32. Naccache, S. N. et al. Diagnosis of neuroinvasive astrovirus infection in an immunocompromised adult with encephalitis by unbiased next-generation sequencing. Clin. Infect. Dis. 60, 919-923 (2015).

33. Lum, S. H. et al. An emerging opportunistic infection: fatal astrovirus (VA1/HMO-C) encephalitis in a pediatric stem cell transplant recipient. Transpl. Infect. Dis. 18, 960-964 (2016).

34. Beck, E. S. et al. Clinicopathology conference: 41-year-old woman with chronic relapsing meningitis. Ann. Neurol. 85, 161-169 (2019).

35. Graus, F. et al. A clinical approach to diagnosis of autoimmune encephalitis. Lancet Neurol. 15 391-404 (2016)

36. Dubey, D. et al. Autoimmune encephalitis epidemiology and a comparison to infectious encephalitis. Ann. Neurol. 83, 166-177 (2018).

37. Pruss, H. et al. Retrospective analysis of NMDA receptor antibodies in encephalitis of unknown origin. Neurology 75, 1735-1739 (2010).

38. Gable, M. S., Sheriff, H., Dalmau, J., Tilley, D. H. $\&$ Glaser, C. A. The frequency of autoimmune $\mathrm{N}$-methyl-D-aspartate receptor encephalitis surpasses that of individual viral etiologies in young individuals enrolled in the California encephalitis project. Clin. Infect. Dis. 54, 899-904 (2012).

39. Leypoldt, F., Wandinger, K. P., Bien, C. G. \& Dalmau, J. Autoimmune encephalitis. Eur. Neurol. Rev. 8, 31-37 (2013).

40. Tobin, W. O. \& Pittock, S. J. Autoimmune neurology of the central nervous system. Continuum 23 . 627-653 (2017)

41. Debiasi, R. L. \& Tyler, K. L. Molecular methods for diagnosis of viral encephalitis. Clin. Microbiol. Rev. 17 903-925 (2004)

42. Hasan, M. R., Tan, R., Al-Rawahi, G. N., Thomas, E. $\&$ Tilley, P. Short-term stability of pathogen-specific nucleic acid targets in clinical samples. J. Clin. Microbiol. 50, 4147-4150 (2012)

43. Brink, M., Welinder-Olsson, C. \& Hagberg, L. Time window for positive cerebrospinal fluid broad-range bacterial PCR and Streptococcus pneumoniae immunochromatographic test in acute bacterial meningitis. Infect. Dis. 47, 869-877 (2015).

44. Zhang, X. X. et al. The diagnostic value of metagenomic next-generation sequencing for identifying Streptococcus pneumoniae in paediatric bacterial meningitis. BMC Infect. Dis. 19, 495 (2019)

45. Hoffmann, B. et al. A variegated squirrel bornavirus associated with fatal human encephalitis. N. Engl. J. Med. 373, 154-162 (2015).

46. Korn, K. et al. Fatal encephalitis associated with Borna disease virus 1. N. Engl. J. Med. 379, 1375-1377 (2018).

47. Schlottau, K. et al. Fatal encephalitic borna disease virus 1 in solid-organ transplant recipients. N. Engl. J. Med. 379, 1377-1379 (2018).

48. Palmiere, C., Egger, C., Prod'Hom, G. \& Greub, G. Bacterial translocation and sample contamination in postmortem microbiological analyses. J. Forensic Sci. 61, 367-374 (2016)

49. Bahr, N. C. et al. GeneXpert MTB/Rif to diagnose tuberculous meningitis: perhaps the first test but not the last. Clin. Infect. Dis. 62, 1133-1135 (2016).

50. Zinter, M. S. et al. Pulmonary metagenomic sequencing suggests missed infections in immunocompromised children. Clin. Infect. Dis. 68, 1847-1855 (2019).

51. Gu, W., Miller, S. \& Chiu, C. Y. Clinical metagenomic next-generation sequencing for pathogen detection. Annu. Rev. Pathol. 14, 319-338 (2019).

52. Charalampous, T. et al. Nanopore metagenomics enables rapid clinical diagnosis of bacterial lower respiratory infection. Nat. Biotechnol. 37, 783-792 (2019).

53. Greninger, A. L. et al. Rapid metagenomic identification of viral pathogens in clinical samples by real-time nanopore sequencing analysis. Genome Med. 7, 99 (2015).

54. Moon, J. et al. Rapid diagnosis of bacterial meningitis by nanopore $16 \mathrm{~S}$ amplicon sequencing: a pilot study. Int. J. Med. Microbiol. 309, 151338 (2019).

55. Hong, N. T. T. et al. Performance of metagenomic next-generation sequencing for the diagnosis of vira meningoencephalitis in a resource-limited setting. Open. Forum Infect. Dis. 7, ofaa046 (2020).

56. MacCannell, D. Platforms and analytical tools used in nucleic acid sequence-based microbial genotyping procedures. Microbiol. Spectr. https://doi.org/10.1128/ microbiolspec.AME-0005-2018 (2019).

57. Langelier, C. et al. Integrating host response and unbiased microbe detection for lower respiratory tract infection diagnosis in critically ill adults. Proc. Natl Acad. Sci. USA 115, E12353-E12362 (2018).

58. Sichtig, $\mathrm{H}$. et al. FDA-ARGOS is a database with public quality-controlled reference genomes for diagnostic use and regulatory science. Nat. Commun. 10, 3313 (2019).

59. Goldberg, B., Sichtig, H., Geyer, C., Ledeboer, N. $\&$ Weinstock, G. M. Making the leap from research laboratory to clinic: challenges and opportunities for next-generation sequencing in infectious disease diagnostics. mBio 6, e01888-15 (2015).

60. Goodacre, N., Aljanahi, A., Nandakumar, S., Mikailov, M. \& Khan, A. S. A reference viral database (RVDB) to enhance bioinformatics analysis of highthroughput sequencing for novel virus detection. mSphere 3, e00069-18 (2018).

61. Zinter, M. S., Mayday, M. Y., Ryckman, K. K. Jelliffe-Pawlowski, L. L. \& DeRisi, J. L. Towards precision quantification of contamination in metagenomic sequencing experiments. Microbiome 7 , 62 (2019)

62. Davis, N. M., Proctor, D. M., Holmes, S. P., Relman, D. A. \& Callahan, B. J. Simple statistical identification and removal of contaminant sequences in marker-gene and metagenomics data. Microbiome 6, 226 (2018)

63. Martelius, T., Lappalainen, M., Palomaki, M. $\&$ Anttila, V. J. Clinical characteristics of patients with Epstein Barr virus in cerebrospinal fluid. BMC Infect. Dis. 11, 281 (2011)

64. Seeley, W. W. et al. Post-transplant acute limbic encephalitis: clinical features and relationship to HHV6. Neurology 69, 156-165 (2007)

65. Doan, T. et al. Illuminating uveitis: metagenomic deep sequencing identifies common and rare pathogens. Genome Med. 8, 90 (2016).

66. Langelier, C. et al. Microbiome and antimicrobial resistance gene dynamics in international travelers. Emerg. Infect. Dis. 25, 1380-1383 (2019).

67. Grubaugh, N. D. et al. Travel Surveillance and Genomics uncover a hidden Zika outbreak during the waning epidemic. Cell 178, 1057-1071.e11 (2019).

68. Crawford, E. et al. Investigating transfusion-related sepsis using culture-independent metagenomic sequencing. Clin. Infect. Dis. https://doi.org/10.1039/ $\mathrm{cid} / \mathrm{ciz} 960$ (2019)

69. GU, W. et al. Depletion of abundant sequences by hybridization (DASH): using Cas9 to remove unwanted high-abundance species in sequencing libraries and molecular counting applications. Genome Biol. 17, 41 (2016).

70. Briese, T. et al. Virome capture sequencing enables sensitive viral diagnosis and comprehensive virome analysis. mBio 6, e01491-01415 (2015).

71. Chalkias, S. et al. ViroFind: a novel target-enrichment deep-sequencing platform reveals a complex JC virus population in the brain of PML patients. PLoS One 13 e0186945 (2018)
72. Quan, J. et al. FLASH: a next-generation CRISPR diagnostic for multiplexed detection of antimicrobial resistance sequences. Nucleic Acids Res. 47, e83 (2019).

73. Deng, X. et al. Metagenomic sequencing with spiked primer enrichment for viral diagnostics and genomic surveillance. Nat. Microbiol. 5, 443-454 (2020).

74. Brown, J. R., Bharucha, T. \& Breuer, J. Encephalitis diagnosis using metagenomics: application of next generation sequencing for undiagnosed cases. J. Infect. 76, 225-240 (2018).

75. Rodriguez, C. et al. Fatal encephalitis caused by cristoli virus, an emerging orthobunyavirus, France. Emerg. Infect. Dis. 26, 1287-1290 (2020).

76. Xing, X. W. et al. Metagenomic next-generation sequencing for diagnosis of infectious encephalitis and meningitis: a large, prospective case series of 213 patients. Front. Cell Infect. Microbiol. 10, 88 (2020).

77. Wang, S. et al. The feasibility of metagenomic nextgeneration sequencing to identify pathogens causing tuberculous meningitis in cerebrospinal fluid. Front. Microbiol. 10, 1993 (2019).

78. Fulton, B. D. et al. Exploratory analysis of the potential for advanced diagnostic testing to reduce healthcare expenditures of patients hospitalized with meningitis or encephalitis. PLoS One 15, e0226895 (2020).

79. Sweeney, T. E., Shidham, A., Wong, H. R. \& Khatri, P. A comprehensive time-course-based multicohort analysis of sepsis and sterile inflammation reveals a robust diagnostic gene set. Sci. Trans/ Med. 7, 287 ra271 (2015).

80. Woods, C. W. et al. A host transcriptional signature for presymptomatic detection of infection in humans exposed to influenza H1N1 or H3N2. PLoS One 8, e52198 (2013).

81. Zaas, A. K. et al. A host-based RT-PCR gene expression signature to identify acute respiratory viral infection. Sci. Transl Med. 5, 203ra1 26 (2013).

82. Tsalik, E. L. et al. Host gene expression classifiers diagnose acute respiratory illness etiology. Sci. Trans I Med. 8, 322ra311 (2016)

83. Holcomb, Z. E., Tsalik, E. L., Woods, C. W. \& McClain, M. T. Host-based peripheral blood gene expression analysis for diagnosis of infectious diseases. J. Clin. Microbiol. 55, 360-368 (2017)

84. Xu, G. J. et al. Viral immunology. Comprehensive serological profiling of human populations using a synthetic human virome. Science 348, aaa0698 (2015).

85. Schubert, R. D. et al. Pan-viral serology implicates enteroviruses in acute flaccid myelitis. Nat. Med. 25 , 1748-1752 (2019).

86. Johnson, T. P. et al. Chronic dengue virus panencephalitis in a patient with progressive dementia with extrapyramidal features. Ann. Neurol. 86, 695-703 (2019).

87. Leon, K. E. et al. Genomic and serologic characterization of enterovirus A71 brainstem encephalitis. Neurol. Neuroimmunol. Neuroinflamm. 7, e703 (2020).

88. Myhrvold, C. et al. Field-deployable viral diagnostics using CRISPR-Cas13. Science 360, 444-448 (2018)

89. Gootenberg, J. S. et al. Multiplexed and portable nucleic acid detection platform with Cas 13, Cas 12a, and Csm6. Science 360, 439-444 (2018).

90. Chen, J. S. et al. CRISPR-Cas 12 a target binding unleashes indiscriminate single-stranded DNase activity. Science 360, 436-439 (2018).

91. Chiu, C. Cutting-edge infectious disease diagnostics with CRISPR. Cell Host Microbe 23, 702-704 (2018)

92. Broughton, J. P. et al. CRISPR-Cas 12-based detection of SARS-CoV-2. Nat. Biotechnol. https://doi.org/ 10.1038/s41587-020-0513-4 (2020).

93. Ackerman, C. M. et al. Massively multiplexed nucleic acid detection using Cas13. Nature 582, 277-282 (2020).

94. Rowley, A. H., Whitley, R. J., Lakeman, F. D. \& Wolinsky, S. M. Rapid detection of herpes-simplexvirus DNA in cerebrospinal fluid of patients with herpes simplex encephalitis. Lancet 335, 440-441 (1990).

95. Leber, A. L. et al. Multicenter evaluation of BioFire filmarray meningitis/encephalitis panel for detection of bacteria, viruses, and yeast in cerebrospinal fluid specimens. J. Clin. Microbiol. 54, 2251-2261 (2016).

96. Clarridge, J. E. III Impact of 16S rRNA gene sequence analysis for identification of bacteria on clinical microbiology and infectious diseases. Clin. Microbiol. Rev. 17, 840-862 (2004).

97. Lindsley, M. D., Hurst, S. F., lqbal, N. J. \& Morrison, C. J. Rapid identification of dimorphic and yeast-like fungal pathogens using specific DNA probes. J. Clin. Microbiol. 39, 3505-3511 (2001). 


\section{REVIEWS}

98. Srinivasan, L., Pisapia, J. M., Shah, S. S., Halpern, C. H. Acknowledgements

$\&$ Harris, M. C. Can broad-range $16 \mathrm{~S}$ ribosomal ribonucleic acid gene polymerase chain reactions improve the diagnosis of bacterial meningitis? A systematic review and meta-analysis. Ann. Emerg. Med. 60, 609-620.e2 (2012).

99. Meyer, T. et al. Improved detection of bacterial central nervous system infections by use of a broadrange PCR assay. J. Clin. Microbiol. 52, 1751-1753 (2014).

100. Solomon, I. H. et al. Fatal Powassan encephalitis (Deer Tick Virus, Lineage II) in a patient with fever and orchitis receiving rituximab. JAMA Neurol. 75, 746-750 (2018)
The authors thank the many committed neurologists and other physicians around the world whose dogged pursuit of diagnoses in patients with complex neuroinflammatory diseases, combined with their collaborative spirit, have led to many of the landmark studies cited in this Review. The authors also thank the patients and families for their participation in the many research studies and families for their participation in the many research studies tions. Lastly, they thank the many government and philanthropic foundations that have supported work in this area.

\section{Author contributions}

Both authors contributed equally to all aspects of the manuscript.
Competing interests

The authors declare no competing interests.

Peer review information

Nature Reviews Neurology thanks Justin McArthur, Paoloa Cinque and the other, anonymous, reviewer(s) for their contribution to the peer review of this work.

\section{Publisher's note}

Springer Nature remains neutral with regard to jurisdictional claims in published maps and institutional affiliations.

(c) Springer Nature Limited 2020 